\title{
25 Research Soure \\ Sporadic utilization of ITN among households with under-five HIV positive children
}

David Ayah Ounah ( $\nabla$ davidounah1@gmail.com )

GLUK https://orcid.org/0000-0002-8915-2873

\section{Jane Mumma}

Great Lakes University of Kisumu

Constantine Loum

Great Lakes University of Kisumu

\section{Susan Amuti}

University of Eldoret

\section{Gideon Kikuvi}

Jomo Kenyatta University of Agriculture and Technology

Nzioki Mativo

Jomo Kenyatta University of Agriculture and Technology

\section{Research article}

Keywords: ITN utilization; Malaria; HIV positive; Under-five Children; Household; Kimilili

Posted Date: November 11th, 2019

DOI: https://doi.org/10.21203/rs.2.17110/v1

License: (c) (i) This work is licensed under a Creative Commons Attribution 4.0 International License.

Read Full License 


\section{Abstract}

Children under-five are most vulnerable and bear the adverse effects of Malaria worldwide. In Kenya, 20\% of all deaths in children are due to malaria, and $88.9 \%$ of all deaths among HIV positive children are malaria related. Correct and consistence ITN utilization is proven to protect against anopheles mosquito bites hence Malaria, however, despite free distribution of ITN to the study population and civic education on proper ITN use, the prevalence of Malaria is still high in this cohort. Therefore, there was an indelible need to establish the levels of household ITN ownership and utilization vis-à-vis demographic characteristics of participants and Malaria attacks among households with HIV positive children in Kimilili. Method: This was a community based study conducted between March and August, 2016 through a cross-sectional survey. The under-five children were recruited randomly at the comprehensive care centre in Kimilili Sub-County Referral Hospital and studied at their households; they were consented through their parents/caretakers. Data on demographic variables, ITN usage and Malaria attacks were collected using questionnaires and analyzed using Statistical Package for Social Scientists, percentages and chi square. Results: The ownership of ITN among the 222 study participants was $98 \%$. The study established that there was poor (88.7\%) household utilization of Insecticide Treated Nets. we further established that among poor ITN users, malaria attacks were high $-65.3 \%$. Conclusion: Utilization of ITN at household level was poor; to the extent that it could not contribute to reduction of malaria associated morbidity and mortality. Efforts should be targeted at households to ensure correct utilization of ITN

\section{Background}

Malaria, a disease caused by Plasmodium but transmitted to humans by female anopheles mosquitoes during blood meal [1] is both treatable and preventable. There are four common species of Plasmodium: Plasmodium falciparum, Plasmodium vivax, Plasmodium ovale and Plasmodium malariae, but in Kenya almost $98 \%$ of infection is caused by Plasmodium falciparum, [2,3].

The epidemiology of Malaria and HIV illustrate geographical overlap, where dual infection is known to play a significant role in fuelling the spread of the two diseases in sub-Saharan Africa [4,5]; in that HIV infection indirectly influence malaria burden by increasing malaria parasite biomass [6], while conversely Malaria worsen HIV by increasing viral load leading to AIDS $[7,8,9,10,11,12]$. Further to this, HIV-1 infected persons are at increased risk of malaria infection than their HIV negative counterparts [13].

Malaria is a major cause of severe Malarial Anemia $(\mathrm{HB}<6 \mathrm{~g} / \mathrm{dl})$ in HIV positive children $[14,15]$. The coexistence of the two diseases consequently makes Malaria a leading killer among HIV positive populations; and since the cure for HIV is not yet proven, prevention of Malaria by proper ITN usage remains one among the best intervention strategies to mitigate against Malaria infection $[10,1,16]$.

Documentation has shown that HIV patients in malaria-endemic areas, who correctly and consistently use ITN, have fewer and less severe episodes of malaria $[17,18,19,20]$. However, Knowledge about appropriate usage of ITNs among HIV positive ITN recipients and their household members are not well 
characterized [21] as is the relationship between malaria and HIV infections in childhood - when most deaths from malaria occur, [22].

The World Health Organization recommends that children less than five years of age in sub-Saharan Africa should have the highest priority for receiving ITNs. However, possession of ITNs in sub-Saharan Africa remains low, with only $6.7 \%$ of households owning one [23] while in Kenya, in $2008,48 \%$ of households owned an ITN, 39\% of children under five had slept under an ITN [24]. Importantly, longlasting ITNs maintain efficacy without re-treatment with insecticide for up to 20 washes [25] making it possible for maintaining cleanliness and hygiene of the netting material without losing potency.

ITN provision to HIV affected individuals in holoendemic communities may serve as a strategy for 'herd protection' if high ITN coverage is achieved in targeted communities [26]. However, the WHO has since established that the Kenyan mosquito has developed resistance to not only some synthetic pyrethroid insecticides, but also against DDT [27,28].

This study aims at collecting a multifaceted baseline data - based on ITN ownership; level of ITN utilization - routine and proper use of ITN, making sure the ITNs are used every night, are well-secured and care taken to avoid mosquitoes entering the ITN; demographic characteristics of study participants; and Malaria attacks. The acuteness and severity of Malaria as manifested by high morbidity and mortality within a short time among the HIV positive under-five year old children makes this study explore prevention of Malaria infection by way of ITN as the most convenient intervention measure.

\section{Methods}

\section{Description of the study area}

This study was household based and was carried out in Kimilili Sub-County which lies in Western Kenya Region which is a Malaria holo-endemic area. The Sub-County has two administrative Divisions, eight administrative locations and 16 administrative sub-locations. The study area has only one major referral health facility with a fully-fledged Comprehensive Care Centre - Kimilili Sub-County Referral Hospital. The hospital is served by small public health facilities located in each of the eight administrative locations plus several other private and mission health facilities. The population of Kimilili Sub-County according to the 2009 housing and population census was 320,300 people of which 155,771 were males and 164,529 females and 101000 under-five children. The population density is 572.44 with 61,486 households. Kimilili has two rainy seasons; long rains from March to June and short rains from September to December [29]. Malaria endemicity peaks up during the long rains season. The annual temperature range of Kimilili is between $18^{\circ} \mathrm{C}$ to $29^{\circ} \mathrm{C}$ with an average humidity of $80 \%$. The main economic activity in the area is agriculture involving farming of coffee, maize, beans, sugarcane, bananas, potatoes plus horticulture crops

\section{Study population}


The study subjects were 222 under-five HIV positive children, randomly and proportionately recruited from eight administrative locations of Kimilili Sub-County. The parents/caretakers/guardians acted on behalf of the under-five children as respondents.

\section{Study design}

The study was a household based cross-sectional survey, conducted between March to August 2016, using a pre-tested structured questionnaire as data collection tool. The researcher and his fellow coresearchers trained a team of three research assistants for one week on how to collect data using the data collection tools, the local cultural norms were adopted in the approach of the respondents' households and the consenting procedure before commencement of face to face interviews with respondents to collect data was undertaken.

Data was collected at a household level and hence we defined a household as: a unit with a married man and his wife/wives plus all of his dependants currently living with him [30]. The under-five parent/caretaker who was a household head was identified and acted as a respondent on behalf of his/her participating under-five child. The researcher and his research assistants outlined in details the purpose of the study to respondents, the rights of participants, benefits and risks associated with the study. The respondents were then allowed an opportunity to either accept to participate in the study through voluntary written informed consent before the questionnaire was administered or alternatively decline to grant consent and hence excluded from the study.

The study questionnaire had two parts; the first part was primarily for collection of demographic variables of both study subjects and respondents (parents/caretakers/guardians) which included age and sex of child; age and sex of respondent, level of education, marital status, occupation and type of house. The second part was designed to capture data on ITN ownership, ITN usage and household Malaria attacks.

A sample size of 226 was calculated using Yemane's formula [31]. However, the response rate among respondents was $98.2 \%$, which translated to only 222 under-five HIV positive children participating in the study.

\section{Sampling method}

Stratified random-sampling technique based on eight administrative locations in Kimilili Sub-County was used to proportionately select under-five HIV positive children to be included in the final study sample. HIV positive status of study subjects was well known in advance, as it was on the basis of their HIV positive status that they were recruited at the Comprehensive Care Centre; they were re-tested and confirmed positive by the Comprehensive Care Centre as described by Rutto et al [7] and hence started on antiretroviral therapy. The randomly selected study participants were traced to their households based on special forms called client household locator forms and records held at Kimilili Sub-County Hospital's Comprehensive Care Centre. These household locator forms and records had details of the names of all children enrolled in Comprehensive Care Centre; names of their parents/caretakers; physical location of 
their homes; Chiefs and Other fine details. Therefore using the locator forms it was easy to trace the eligible study homes.

\section{Ethical considerations}

Ethical clearance was sought from Great Lakes University of Kisumu (GLUK) Research Ethics Review Committee and Certificate of Approval of Research Protocol was granted under reference number, GREC 065/11/2012.

The hospital and local administration gave consent to the study. The research respondents granted written informed consent on behalf of their participating under-five year old children. The consenting process was done using Informed Consent Agreement Form, that were available in both English and local

Luhya languages, therefore respondents were consented in the languages of their choices and which they understood best.

The study ensured the coding of its participants data and that their identity remained anonymous from the time of recruitment into the study up to the conclusion of the study. All information relating to study subjects was kept confidential with no access to third parties.

The research tools were pre-tested to guarantee accuracy and validity before being used.

\section{Statistical analysis}

Data was collected directly on the study questionnaires, checked for validity and entered onto computer excel spreadsheet by two data entry clerks. This was then merged into one spreadsheet by a third clerk and inconsistences corrected using original data collection questionnaires.

The excel spreadsheet was then scored for each primary variable of investigation, rated and coded manually. The data was exported into Statistical Package for Social Sciences (SPSS) version 11.5 and analyzed. Chi-square and $95 \%$ confidence interval analysis were used as statistical tools to determine statistical significance to declare an association between different variables at a cut-off value of $95 \%$ ( $P$ $=0.05)$. The results were presented in frequencies, percentages and descriptive statistics.

The appropriate responses which included routine and consistency use of ITN implied correct utilization of ITN at household level; this was rated good and coded 1. The responses that were a mixture of both haphazard and consistency ITN utilization implied median ITN use and were rated fair and coded 2; while the inappropriate responses which implied haphazard and inconsistency use of ITNs were scored low, rated poor and coded 3 .

\section{Limitations of the study}

The following were the major limitaions of this study; Firstly, the study was localized in Kimilili SubCounty yet Malaria is a national and a global problem. A study of this kind should have covered at least a whole region to justify inference of its findings to general population. Secondly, This study may have 
also been limited by recall bias in its attempt to establish the facts as to the utilization of ITN without misqivings. Not all respondents may have clearly recalled how they utilized ITN during administration of the questionnaire. Thirdly, the validity and accuracy of tools of data collection may not have been $100 \%$ guaranteed despite having been pre-tested and validated during pilot study.

\section{Results}

Table 4.1 Background characteristics of study subjects and respondents Study subject

\section{Percent}

1. Age of child in months

2. Gender of child

Study respondent

1. Age in years

2. Type of respondent

3. Gender

4. Marital status

5. Education

6. Occupation

7. Status of mother

8. Status of father
$(0-12$

months)

(27.9\%)

Girls (53.2\%)

21-30 years

(36.5\%)

Mother

(91.0\%)

Female

(98.2\%)

Married

(53.2\%)

Primary

(31.5\%)

Formal job

(27.5\%)

Alive

(96.4\%)

Alive

(93.2\%)
(13 -24 months)

(27.0\%)

Boys (46.8\%)

31-40 years

(31.5\%)

Father (1.8\%)

Male (1.8\%)

Separated

(22.1\%)

Secondary

(29.3\%)

Business (24.8\%)

Deceased (3.2\%)

Deceased (6.8\%)
41-50 years

(23.9\%)

Other (7.2\%)

(25 - 36

months)

(30.2\%)

(37 - 48

months)

(14.9\%)

-

$<20 \& 50+$
years

(9.1\%)

Single

(16.7\%)

Tertiary

(31.5\%)

Farming

(14.9\%)

Other/unknown

(0.5\%)
Widowed

(6.8\%)

Univ./none

(7.7\%)

Other/Jobless

(32.9\%)

Table 4.1 shows that under-five children of all ages and either gender were captured in almost equal proportions at an average of $25.0 \%$ and $50.0 \%$ respectively. The majority $(91.0 \%)$ of caretakers of these children was biological mothers - thus females, $1.8 \%$ were fathers and $7.2 \%$ were mainly aunts, step mothers, house helps grandparents and other relatives.

Most (36.5\%) respondents were in their reproductive age bracket of between ( $21-40 \mathrm{~s})$ years old with a cumulative percentage of $68.0 \%$ and of these only $53.2 \%$ were married.

Those respondents with formal education had a cumulative percentage of $96.8 \%$, and $3.2 \%$ had no formal education. Consequently, $68.1 \%$ were engaged in income generating activity with $31.9 \%$ being 
jobless. Status of parents showed $96.4 \%$ and $93.2 \%$ of under-five children had their biological mothers and fathers alive respectively.

\section{ITN utilization}

ITN utilization was a core component in this study as correct utilization of ITN at household level was one of the elements of malaria prevention and control health package. The respondents were requested to react to several questions intended to measure their way of utilization of ITNs. Depending on the responses, the utilization of ITN in each household was rated as good, fair or poor.

The number of under-five HIV positive children who had been attacked by malaria in each household were also established and then grouped under each category of household utilization level of ITN. The results of the analysis were summarized in Table 4.2.

Table 4.2 ITN utilization and malaria attack among households with under-five HIV positive children

\begin{tabular}{lllll}
\hline $\begin{array}{c}\text { ITN Utilization } \\
\text { Rating/Levels }\end{array}$ & & \multicolumn{2}{l}{ Malarial Attack } & Household ITN \\
\cline { 3 - 4 } Utilization \\
\hline Poor/incorrect & $\mathrm{N}$ & 145 & 52 & 197 \\
& & $65.3 \%$ & $23.4 \%$ & $88.7 \%$ \\
\hline Fair/less-correct & $\mathrm{N}$ & 17 & 8 & 25 \\
& & $7.7 \%$ & $3.6 \%$ & $11.3 \%$ \\
\hline Good/correct & $\mathrm{N}$ & 0 & 0 & 0 \\
& & $0.0 \%$ & $0.0 \%$ & $0.0 \%$ \\
\hline Total & $\mathrm{N}$ & 162 & 60 & 222 \\
& & $73.0 \%$ & $27.0 \%$ & $100.0 \%$ \\
\hline
\end{tabular}

Note. $N=$ frequency of households

Table 4.2 shows that no single household reported correct ITN utilization of ITN, only a few households $25(11.33 \%)$ registered a fair ITN utilization. On overall, ITN utilization was incorrect among 197 (88.7\%) households.

Malaria attack was highest in households where ITN utilization was poor, with 145 (65.3\%) households reporting malaria attacks in under-five HIV positive children and where ITN utilization was fair, malaria attack was only17 (7.7\%). However, no household was found to display a good ITN utilization and therefore we did not establish malaria attacks at this level of ITN utilization.

This tends to show that the increase in malaria among the under-five HIV positive children in Kimilili might be because of poor utilization of ITN component of malaria prevention and control health package. 
The data was further tested using chi square test of independence to establish whether there were significant statistical association between prevalence of malaria attacks and level of household ITN utilization. The results of the chi square analysis were summarized in Table 4.3.

Table 4.3 Results of $\chi^{2}$ Analysis of Prevalence of Malarial attacks based on ITN Utilization

\begin{tabular}{lllllll}
\hline Variable & $\mathrm{n}$ & $\chi_{o}{ }^{2}$ & $\mathrm{df}$ & $X_{C}{ }^{2}$ & $\alpha$ & Decision \\
\hline $\begin{array}{l}\text { Prevalence of malaria attacks versus ITN } \\
\text { utilization. }\end{array}$ & 222 & 2.632 & 2 & 5.990 & .352 & $\begin{array}{l}\text { No } \\
\text { P }\end{array}$ \\
\hline
\end{tabular}

Table 4.3 shows that there was NO significant statistical association in the prevalence of malaria attacks in households with under-five HIV positive children based on level of ITN utilization. The results show that $\chi_{o}^{2}=2.632<X_{c}^{2}=5.990 ;$ and $a_{o}=.352>.05$. The study therefore established that prevalence of malaria attacks among households with under-five year old HIV positive children in Kimilili is independent on ITN utilization.

\section{Discussion}

ITN utilization was a core component in this study as its level of utilization at household influenced malaria attack. Fair utilization of ITN was recorded among $11.3 \%$ of households while poor utilization of ITN was recorded in $88.7 \%$. No household recorded good utilization. The utilization levels of ITN however did not have a significant statistical association with malaria attacks. The households where ITN utilization was fair recorded $7.7 \%$ incidences of malaria attack in the under-five while those with poor ITN utilization recorded the highest incidences of malaria attack at $65.3 \%$. Though there was no good ITN utilization recorded, the findings shows that correct household utilization of ITN is important in reducing malaria attacks. This finding is in line with the findings of the study by Phillip-Howard [16] which found out that ITN usage reduced malaria incidences by $27 \%$. These findings are vital indicators of how ITN can be utilized at household level to successfully reduce malaria burden in vulnerable HIV population of under-five. However, findings by most of other related studies fell short of determining ITN beyond mere coverage. In this study, we established not only ITN coverage but correct utilization at the very consumption point (household) too. This was possible because we recorded $100 \%$ ITN coverage in all study households and accordingly we proved that possession of ITN alone could not rule out malaria infection. Hawley [26] argues that such complete coverage could provide herd protection, which we agree but only if it is coupled with good utilization. This same argument again defeats the fact by Sylvia Becker-Dreps [23], that in sub-Saharan Africa only $6.7 \%$ of households own ITN; Abdisalan [1] states that ITN coverage across Africa is as low as $5 \%$ and another finding by World Malaria Report, 2009 recorded $48 \%$ ITN ownership in Kenya. The scenario on low ITN coverage could have changed because of the current effect of WHO 2007 [17] guidelines that promoted low-cost or free distribution of ITNs for all children and HIV-positive persons in all malaria endemic areas; the impact of this effect is being felt now 
and this contributed to wide ITN coverage, though we have shown that coverage on one hand and good ITN utilization on the other hand are two different things, they must go hand in hand. This study agrees with the findings of both Muema [22] and Lauren [21], that Knowledge about malaria prevention in HIV and appropriate utilization of ITNs among HIV positive ITN recipients and their household members is sparse and has not been well characterized hence leading to poor ITN utilization. The other critical finding of this study is that in some households mosquitoes were reported anchoring on the ITN and this pointed to the fact that though nets were treated with insecticides, the potency of these insecticides was very low to immobilise mosquitoes found resting on ITNs or mosquitoes could have developed resistance to these insecticides. This finding is in line with findings of Corbel [27] and KDHS [28] which reported a $25 \%$ increase in malaria infections due to mosquito resistance to insecticides used in treating the nets; however the finding contradicts Giming [25] proposition that ITNs maintain efficacy without insecticide re-treatment for up to 20 washes.

\section{Conclusion}

This study found out that the correct utilization of ITN in malaria prevention was deficient in Kimilili SubCounty. Most households in the division had poor utilization of ITNs and only few had fair utilization yet correct utilization had been shown in previous studies to reduce malaria attacks; however, there was no household that recorded correct utilization of ITN in this study. Therefore high prevalence of malaria in this area was partly attributable to poor utilization of ITN at household level; to reverse this, ITNs should be utilized correctly as postulated by MPC package.

\section{Abbreviations}

ITNs: Insecticide-treated bed nets; HIV: Human immunodeficiency Virus.

\section{Declarations}

\section{Competing interests}

The authors declare that they have no financial and/or personal competing interests that may inappropriately influence them in writing this article.

\section{Authors' contributions}

DAO designed the study, conducted the research work, analyzed the data, wrote the draft and submitted the final manuscript. JM \& CL supervised the study design and implementation. GK, NM, JO \& SA read the draft and made corrections on Methodology. All authors read, edited and approved the final manuscript.

\section{Authors' information}


DAO: PhD Candidate in Public Health, Department of Public Health, Jomo Kenyatta University of Agriculture and Technology University. JM: PhD and Lecturer of Community Health - Great Lakes University of Kisumu. CL: PhD and Lecturer of Community Health - Great Lakes University of Kisumu. SA: PhD, Lecturer Department of Microbiology, University of Eldoret. JO: PHD, Maseno University. GK: PHD, Professor of Public Health, Jomo Kenyatta University of Agriculture and Technology University, NM: Lecturer Public Health, Jomo Kenyatta University of Agriculture and Technology University

\section{Acknowledgements}

We are sincerely grateful to all research study subjects and respondents who voluntarily participated in this study. We acknowledge as well the invaluable input of Prof. Dan Kaseje - The Vice Chancellor of Great Lakes University of Kisumu. Prof. Kaseje could spare time from his busy schedule, to personally review the final research thesis successfully - many thanks indeed Prof. I am also indebted to Damaris Nelima for their great input and timely supervision. Thanks to my mum Dolphine Ounah for encouragement. Thanks to the Medical Superintendent of Kimilili Sub-County Hospital, Dr. Wanikina and staff for their invaluable support. Kudos to Rev. Obondi, Dr. Otieno and Dr. Osoo for working with me on general Masters Thesis which is the basis for this publication. Lastly thanks to my family for standing with me - Pauline, Sylvia, Rossie, Gloria, Benja, Khatuyu, Martha, Mitchelle, Manea and Claire. This work was supported by the special fund for research and modernization given to authors by the Great Lakes University of Kisumu.

\section{References}

1. Abdisalan M. N., Juliette J. M., Andrew J. T., Simon I. H., and Snow W. (2009). Insecticide-treated net coverage in Africa: mapping progress in 2000-07. Lancet, Vol. 373(9657): 58-67.

2. Ernest Tambo, Ahmed Adebowale Adedeji, Fang Huang, Jun-Hu Chen, Shui-Sen Zhou ${ }^{1}$ and Ling-Hua Tang ; Scaling up impact of malaria control programmes: a tale of events in Sub-Saharan Africa and People's Republic of China; Infectious Diseases of Poverty 2012, 1:7 doi:10.1186/2049-9957-1-7; http://www.idpjournal.com/content/1/1/7

3. Maxmen A. A., (2011) Outdoor Mosquitoes Could Defy Control, Nature News

4. Cuadros D.F., Branscum A.J., García-Ramos G. (2011), No evidence of association between HIV-1 and malaria in populations with low HIV-1 prevalence. PLoS One. Vol;6(8):e23458.

5. Diego F Cuadros , Adam J Branscum and Philip H Crowley ; HIV-malaria co-infection: effects of malaria on the prevalence of HIV in East sub-Saharan Africa; 2010 International Journal of Epidemiology; Volume 40, Issue 4, Pp. 931-939.

6. Abu-Raddad L.J., Patnaik P., Kublin J.G. (2006). Dual infection with HIV and malaria fuels the spread of both diseases in sub-Saharan Africa.Science, 314:1603-1606.

7. Erick Kipkoech Rutto, Joshua Nyagol, Julius Oyugi, Samson Ndege, Noel Onyango, Andrew Obala, Chrispinus J Simiyu, Gye Boor, Winfrida Chelangat Cheriro, Barasa Otsyula and Ben Estambale; Effects of HIV-1 infection on malaria parasitemia in milo sub-location, western Kenya; BMC Research 
Notes 2015, 8:303 doi:10.1186/s13104-015-1270-1; http://www.biomedcentral.com/17560500/8/303

8. Alemu A, Shiferaw Y, Addis Z, Mathewos B, Birhan W: Effect of malaria on HIV/AIDS transmission and progression. Parasit Vectors 2013, 6:

9. Iroezindu M, Agaba E, Daniyam C, Okeke E, Agbaji O, Agaba P, et al:: Association of HIV-induced immunosuppression and clinical malaria in Nigerian adults. Afr J Infect Dis 2012, 6(2):48-53.

10. World Health Organization. 2010, Combating HIV/AIDS, malaria and other diseases, Geneva: World Health Organization.

11. Kamya M.R., Gasasira A.F., Achan J., Mebrahtu T., Ruel T., Kekitiinwa A., Charlebois E.D., Rosenthal P.J., Havlir D., Dorsey G. (2007), Effects of trimethoprim-sulfamethoxazole and insecticide-treated bednets on malaria among HIV-infected Ugandan children. Vol. 1;21(15):2059-66.

12. Kamya, Moses R.; Kigonya, Catherine N.; McFarland, Willi; HIV infection may adversely affect clinical response to chloroquine therapy for uncomplicated malaria in children; AIDS: 15 June 2001 - Volume 15 - Issue 9 - pp 1187-1188

13. Maganda BA, Minzi OM, Kamuhabwa AA, Ngasala B, Sasi PG: Outcome of artemether-lumefantrine treatment for uncomplicated malaria in HIV-infected adult patients on anti-retroviral therapy. Malar $J$ 2014, 13(1):205.

14. World Health Organization: World Malaria Report. Switzerland: World Health Organization; 2012.

15. Otieno, Richard O; Ouma, Collins; Ong'echa, John M; Keller, Christopher C; Were, Tom; Waindi, Eliud N; Michaels, Marian G; Day, Richard D; Vulule, John M; Perkins, Douglas J; Increased severe anemia in HIV-1-exposed and HIV-1-positive infants and children during acute malaria; AIDS: 9 January 2006 Volume 20 - Issue 2 - p 275-280; doi:10.1097/01.aids.0000200533.56490.b7

16. Phillip-Howard P.A., Nahlen B.L., Kolesak M.S., Hightower A.W., TerKuile F.O., Alaii J.A., Gimning, J.E., Vulule J.M., Odhacha, a., Kachur, P.S., Schoute, e., Rosen, D.H., Sexton, J.D., Oloo, A.J., Hawley, W.A. (2003). Efficacy of permethrin treated bed nets in the area of high malaria transmission in western Kenya. American journal of tropical medicine and hygiene, Vol. 68 (4); 23-29.

17. World Health Organization. 2007, WHO releases new guidance on insecticide-treated mosquito nets, Geneva/Nairobi: World Health Organization

18. Lengeler C: Insecticide-treated bednets and curtains for preventing malaria; Cochrane Database Syst Rev 2004, 2:1-57; doi:10.1002/14651858.CD000363.pub2. Art. No.: CD000363

19. TerKuile FO, Terlouw DJ, Phillips-Howard PA, Hawley WA, Friedman JF, Kariuki SK, Shi YP, Kolezak MS, Lal AA, Vulule JM, Nahlen BL: Reduction of malaria during pregnancy by permethrin-treated bed nets in an area of intense perennial malaria transmission in western Kenya. Am J Trop Med Hyg 2003, 64(2 Suppl):50-60.

20. Hill J, Lines J, Rowland M: Insecticide-treated nets. Adv Parasito/ 2006, 61:77-128.

21. Lauren C., Lisa A. M., Kagaayi J., Ilana J., Galiwango R., Ludigo J., Ssekasanvu J.and Steven J. R. (2009), High retention and appropriate use of insecticide-treated nets distributed to HIV-affected 
households in Rakai, Uganda: results from interviews and home visits. Malaria Journal, Vol. 8: 76

22. Muema D.K.M., Ndungu F.M., Kinyanjui S.M. and Berkley J.A. (2011). Effect of HIV infection on the acute antibody response to malaria antigens in children: an observational study. Malaria Journal, doi:10.1186/1475-2875-10-55

23. Sylvia I. Becker-Dreps, Andrea K. B., Audrey P., Gertrude M., David N. I., Steven M. and Frieda B. (2009). Cost-Effectiveness of Adding Bed Net Distribution for Malaria Prevention to Antenatal Services in Kinshasa, Democratic Republic of the Congo.American journal of tropical medicine and hygiene, Vol. 81(3): 496-502

24. World Malaria Report, 2009

25. Gimnig J, Lindblade K.A., Mount D.L., Atieli F.K., Crawford S., Wolkon A., Hawley W.A., Dotson E.M., (2005), Laboratory wash resistance of long-lasting insecticidal nets. Tropical Medecine International Health. Vol. 10: 1022-1029.

26. Hawley W.A., Phillips-Howard P.A., Kuile F.O., Terlouw D.J., Vulule J.M., Ombok M., Nahlen B.L., Gimnig J.E., Kariuki S.K., Kolczak M.S., Hightower A.W., (2003), Community-wide effects of permethrin-treated bed nets on child mortality and malaria morbidity in western Kenya. American Journal of Tropical Medicine Hygiene 68 (4Suppl):121-127.

27. Corbel V., Daily Nation - DN2 2011. Little parasites that have kept scientists on their toes, March 30, pages $2-3$

28. Kenya Demographic Health Survey, 2008-09.

29. Kenya National Bureau of Statistics (KNBS), 2018

30. Helen K Kimbi, Sarah B Nkesa, Judith L Ndamukong-Nyanga, Irene UN Sumbele, Julius Atashili and Mary BS Atanga, Knowledge and perceptions towards malaria prevention among vulnerable groups in the Buea Health District, Cameroon; BMC Public Health 2014, 14:883 doi:10.1186/1471-2458-14883; http://www.biomedcentral.com/1471-2458/14/883

31. Yamane, T. (1967). Statistics: An Introductory Analysis. New York: Harper \& Row

\section{Supplementary Files}

This is a list of supplementary files associated with this preprint. Click to download.

- QUESTIONNAIRE.pdf

- ITNMCHDDatasheetf2019.xIsx 